\title{
Uniqueness of Entire Functions concerning Difference Operator
}

\author{
Chun Wu \\ College of Mathematics Science, Chongqing Normal University, Chongqing 401331, China \\ Correspondence should be addressed to Chun Wu; xcw919@gmail.com \\ Received 1 July 2013; Accepted 27 September 2013 \\ Academic Editor: Marco Sabatini \\ Copyright (C) 2013 Chun Wu. This is an open access article distributed under the Creative Commons Attribution License, which \\ permits unrestricted use, distribution, and reproduction in any medium, provided the original work is properly cited.
}

We deal with a uniqueness question of entire functions sharing a nonzero value with their difference operators and obtain some results, which improve the results of Qi et al. (2010) and Zhang (2011).

\section{Introduction and Main Results}

In this paper, a meromorphic function will mean meromorphic in the whole complex plane. We will use the standard notations of Nevanlinna's value distribution theory such as $T(r, f), N(r, f), \bar{N}(r, f)$, and $m(r, f)$, as explained in Hayman [1], Yang [2], and Yang and Yi [3]. We denote by $S(r, f)$ any quantity satisfying $S(r, f)=o(T(r, f))$, as $r \rightarrow \infty$ possibly outside a set of finite linear measures. For $f$ meromorphic in $\mathbb{C}$, denote by $S(f)$ the family of all meromorphic functions $a(z)$ that satisfy $T(r, a)=o(T(r, f))$ for $r \rightarrow \infty$ outside a possible exceptional set of finite linear measure. In addition, we denote by $\rho(f)$ and $\rho_{2}(f)$ the order of $f$ and the hyperorder of $f[3,4]$. Moreover, we define difference operators by $\Delta_{c} f=f(z+c)-f(z)$ where $c$ is a nonzero constant. If $c=1$, we use the usual difference notation $\Delta_{c} f=\Delta f$.

Let $f$ and $g$ be two nonconstant meromorphic functions and $a$ be a finite complex number. We say that $f, g$ share the value $a \mathrm{CM}$ (counting multiplicities) if $f, g$ have the same $a$ points with the same multiplicities, and we say that $f, g$ share the value $a$ IM (ignoring multiplicities) if we do not consider the multiplicities. We denote by $\bar{N}_{L}(r, 1 /(f-a))$ the counting function for $a$-points of both $f$ and $g$ about which $f$ has larger multiplicity than $g$, with multiplicity not being counted. Similarly, we have the notation $\bar{N}_{L}(r, 1 /(g-a))$. Next, we denote by $N_{0}\left(r, 1 / F^{\prime}\right)$ the counting function of those zeros of $F^{\prime}$ that are not the zeros of $F(F-1)$ and denote by $N_{11}(r, 1 /(f-a))$ the counting function for common simple 1-point of both $f$ and $g$. In addition, we need the following three definitions.

Definition 1. Let $k$ be a positive integer. Let $f$ and $g$ be two nonconstant meromorphic functions such that $f$ and $g$ share the value 1 IM. Let $z_{0}$ be a 1-point of $f$ with multiplicity $p$ and a 1-point of $g$ with multiplicity $q$. We denote by $\bar{N}_{f>k}(r, 1 /(g-$ 1)) the reduced counting function of those 1-points of $f$ and $g$ such that $p>q=k \cdot \bar{N}_{g>k}(r, 1 /(f-1))$ is defined analogously.

Definition 2 (see [5]). Let $k$ be a nonnegative integer or infinity. For $a \in \mathbb{C} \cup \infty$, we denote by $E_{k}(a, f)$ the set of all $a$-points of $f$, where an $a$-point of multiplicity $m$ is counted $m$ times if $\leq k$ and $k+1$ times if $m>k$. If $E_{k}(a, f)=E_{k}(a, g)$, we say that $f, g$ share the value a with weight $k$.

The definition implies that if $f, g$ share a value a with weight $k$, then $z_{0}$ is an $a$-point of $f$ with multiplicity $m(\leq k)$ if and only if it is an $a$-point of $g$ with multiplicity $m(\leq k)$ and $z_{0}$ is an $a$-point of $f$ with multiplicity $m(>k)$ if and only if it is an $a$-point of $g$ with multiplicity $n(>k)$, where $m$ is not necessarily equal to $n$.

We write that $f, g$ share $(a, k)$ to mean that $f, g$ share the value a with weight $k$. Clearly if $f, g$ share $(a, k)$, then $f, g$ share $(a, p)$ for any integer $p, 0 \leq p<k$. Also we note that $f$, $g$ share a value $a$ IM or CM if and only if $f, g$ share $(a, 0)$ or $(a, \infty)$, respectively.

Definition 3. Let $f$ be a nonconstant meromorphic function, and let $p$ be a positive integer and $a \in C \cup\{\infty\}$. Then, by 
$N_{p)}(r, 1 /(f-a))$, we denote the counting function of those $a$ points of $f$ (counted with proper multiplicities) whose multiplicities are not greater than $p$, and by $\bar{N}_{p)}(r, 1 /(f-a))$, we denote the corresponding reduced counting function (ignoring multiplicities). By $N_{(p}(r, 1 /(f-a))$, we denote the counting function of those $a$-points of $f$ (counted with proper multiplicities) whose multiplicities are not less than $p$, and by $\bar{N}_{(p}(r, 1 /(f-a))$, we denote the corresponding reduced counting function (ignoring multiplicities), where $N_{p)}(r, 1 /(f-a)), \bar{N}_{p)}(r, 1 /(f-a)), N_{(p}(r, 1 /(f-a))$, and $\bar{N}_{(p}(r, 1 /(f-a))$ mean $N_{p)}(r, f), \bar{N}_{p)}(r, f), N_{(p}(r, f)$, and $\bar{N}_{(p}(r, f)$, respectively, if $a=\infty$.

In 2010, Qi et al. [6] proved the following uniqueness theorem.

Theorem A. Let $f$ and $g$ be transcendental entire functions of finite order, let $c$ be a nonzero complex constant, and let $n \geq 6$ be an integer. If $f(z)^{n} f(z+c)$ and $g(z)^{n} g(z+c)$ share $z C M$, then $f=\operatorname{tg}$ for a constant that satisfies $t^{n+1}=1$.

In 2011, Zhang et al. [7] complemented the above theorem and obtained the following result.

Theorem B. Let $f$ and $g$ be nonconstant entire functions of finite order, and let $n \geq 5$ be an integer. Suppose that $c$ is a nonzero complex constant such that $\Delta_{c} f \neq \equiv 0$ and $\Delta_{c} g \neq \equiv 0$. If $f^{n} \Delta_{c} f$ and $g^{n} \Delta_{c} g$ share $z C M$, and $g(z+c)$ and $g(z)$ share 0 $C M$ then $f=t g$, where $t$ is a constant satisfying $t^{n+1}=1$.

In this paper, we complement Theorems A and B and obtain the following results which generalize the above theorems.

Theorem 4. Let $f$ be a transcendental entire function of finite order and $\Delta_{c} f \not \equiv 0$, let $a \neq 0$ be a small function with respect to $f$, and let $c$ be a nonzero complex constant. Then for $n \geq 2$, $f(z)^{n}(f(z+c)-1) \Delta_{c} f-a$ has infinitely many zeros.

Theorem 5. Let $f(z)$ and $g(z)$ be transcendental entire functions of $\rho_{2}<1, n \geq 2 k+7$. Suppose that $c$ is a nonzero complex constant such that $\Delta_{c} f \not \equiv 0$ and $\Delta_{c} g \neq 0$. If $\left[f^{n} \Delta_{c} f\right]^{(k)}$ and $\left[g^{n} \Delta_{c} g\right]^{(k)}$ share $1 C M$, then $f=$ tg for a constant $t$ with $t^{n+1}=1$.

Theorem 6. Let $f$ and $g$ be transcendental entire functions of $\rho_{2}<1, n \geq 5 k+13$. $c$ is a nonzero complex constant such that $\Delta_{c} f \neq \equiv 0$ and $\Delta_{c} g \not \equiv 0$. If $\left[f^{n} \Delta_{c} f\right]^{(k)}$ and $\left[g^{n} \Delta_{c} g\right]^{(k)}$ share 1 $I M$, then $f=\operatorname{tg}$ for a constant $t$ with $t^{n+1}=1$.

\section{Some Lemmas}

Lemma 7 (see [8]). Let $f$ be a nonconstant meromorphic function of finite order $\sigma$, and let $c$ be a nonzero constant. Then, for each $\varepsilon>0$,

$$
T(r, f(z+c))=T(r, f(z))+O\left(r^{\sigma-1+\varepsilon}\right)+O(\log r) .
$$

Lemma 8 (see [9]). Let $f$ be a meromorphic function of finite order, and let $c \in \mathbb{C}$ and $\delta \in(0,1)$. Then

$$
\begin{gathered}
m\left(r, \frac{f(z+c)}{f(z)}\right)+m\left(r, \frac{f(z)}{f(z+c)}\right) \\
=o\left(\frac{T(r, f)}{r^{\delta}}\right)=S(r, f) .
\end{gathered}
$$

Lemma 9 (see [10]). Let $f_{1}, f_{2}$, and $f_{3}$ be nonconstant meromorphic functions such that $f_{1}+f_{2}+f_{3}=1$. If $f_{1}, f_{2}$, and $f_{3}$ are linearly independent, then

$$
T\left(r, f_{1}\right) \leq \sum_{j=1}^{3} N_{2}\left(r, \frac{1}{f_{j}}\right)+\sum_{j=1}^{3} \bar{N}\left(r, f_{j}\right)+o(T(r)),
$$

where $T(r)=\max _{1 \leq j \leq 3} T\left(r, f_{j}\right), r \notin E$, and $E$ denote a set of positive real numbers of finite linear measure.

Lemma 10. Let $f$ be transcendental entire functions of finite order, let $c$ be a nonzero complex constant, and set $F(z)=$ $f(z)^{n} \Delta_{c} f ;$ then

$$
n T(r, f)+S(r, f) \leq T(r, F) \leq(n+1) T(r, f)+S(r, f) .
$$

Proof. Since

$$
\begin{aligned}
T(r, F)= & T\left(r, f(z)^{n} \Delta_{c} f\right) \leq n T(r, f)+T\left(r, \Delta_{c} f\right) \\
\leq & n T(r, f)+m\left(r, \Delta_{c} f\right) \leq n T(r, f) \\
& +m(r, f)+S(r, f) \\
= & (n+1) T(r, f)+S(r, f),
\end{aligned}
$$

then

$$
\begin{aligned}
(n+1) T(r, f)= & T\left(r, f(z)^{n+1}\right)=m\left(r, f(z)^{n+1}\right) \\
\leq & m\left(r, \frac{f(z)^{n+1}}{F}\right)+m(r, F)+S(r, f) \\
\leq & m\left(r, \frac{f(z)}{\Delta_{c} f}\right)+m(r, F)+S(r, f) \\
\leq & T\left(r, \frac{f(z)}{\Delta_{c} f}\right)+T(r, F)+S(r, f) \\
\leq & T\left(r, \frac{\Delta_{c} f}{f(z)}\right)+T(r, F)+S(r, f) \\
= & m\left(r, \frac{\Delta_{c} f}{f(z)}\right)+N\left(r, \frac{\Delta_{c} f}{f(z)}\right) \\
& +T(r, F)+S(r, f) \\
\leq & T(r, F)+N\left(r, \frac{1}{f(z)}\right)+S(r, f) \\
\leq & T(r, F)+T(r, f)+S(r, f) .
\end{aligned}
$$


That is,

$$
n T(r, f)+S(r, f) \leq T(r, F) \leq(n+1) T(r, f)+S(r, f) .
$$

Lemma 11 (see [11]). Let $f_{1}$ and $f_{2}$ be two nonconstant meromorphic functions. If $c_{1} f_{1}+c_{2} f_{2}=c_{3}$, where $c_{1}, c_{2}$, and $c_{3}$ are nonzero constants, then

$$
T\left(r, f_{1}\right) \leq \bar{N}\left(r, f_{1}\right)+\bar{N}\left(r, \frac{1}{f_{1}}\right)+\bar{N}\left(r, \frac{1}{f_{2}}\right)+S\left(r, f_{1}\right) .
$$

Lemma 12 (see [12]). Let $f(z)$ be a nonconstant meromorphic function, and let $k$ be a positive integer. Suppose that $f^{(k)} \neq \equiv$; then

$$
N\left(r, \frac{1}{f^{(k)}}\right) \leq N\left(r, \frac{1}{f}\right)+k \bar{N}(r, f)+S(r, f) .
$$

Lemma 13 (see [13]). Let $f$, g share $(1,0)$. Then

(i) $\bar{N}_{f>1}(r, 1 /(g-1)) \leq \bar{N}(r, 1 / f)+\bar{N}(r, f)-N_{0}\left(r, 1 / f^{\prime}\right)+$ $S(r, f)$,

(ii) $\bar{N}_{g>1}(r, 1 /(f-1)) \leq \bar{N}(r, 1 / g)+\bar{N}(r, g)-N_{0}\left(r, 1 / g^{\prime}\right)+$ $S(r, g)$.

Lemma 14. Let $f(z)$ and $g(z)$ be two nonconstant entire functions. If $f$ and $g$ share $1 \mathrm{IM}$, then one of the following cases holds:

(i) $T(r, g) \leq N_{2}(r, 1 / g)+N_{2}(r, 1 / f)+\bar{N}(r, 1 / f)+$ $2 \bar{N}(r, 1 / g)+S(r, f)+S(r, g)$, the same inequality holding for $T(r, f)$;

(ii) $f \equiv(A g+B) /(C g+D)$, where $A, B, C$, and $D$ are finite complex numbers satisfying $A D \neq B C$.

Proof. Let

$$
\Phi(z)=\frac{f^{\prime \prime}}{f^{\prime}}-2 \frac{f^{\prime}}{f-1}-\frac{g^{\prime \prime}}{g^{\prime}}+2 \frac{g^{\prime}}{g-1} .
$$

Clearly $m(r, \Phi)=S(r, f)+S(r, g)$. We consider the cases $\Phi(z) \not \equiv 0$ and $\Phi(z) \equiv 0$.

If $\Phi(z) \not \equiv 0$, then if $z_{0}$ is a common simple 1-point of $f^{\prime}$ and $g^{\prime}$, substituting their Taylor series at $z_{0}$ into (10), we see that $z_{0}$ is a zero of $\Phi(z)$. Thus, we have

$$
\begin{aligned}
N_{11}\left(r, \frac{1}{f-1}\right) & =N_{11}\left(r, \frac{1}{g-1}\right) \leq \bar{N}\left(r, \frac{1}{\Phi}\right) \\
& \leq T(r, \Phi)+O(1) \\
& \leq N(r, \Phi)+S(r, f)+S(r, g) .
\end{aligned}
$$

Our assumptions are that $\Phi(z)$ has poles; all are simple only at zeros of $f^{\prime}$ and $g^{\prime}$ and poles of $f$ and $g$, and 1-points of $f$ whose multiplicities are not equal to the multiplicities of the corresponding 1-points of $g$. Thus, we deduce from (10) that

$$
\begin{aligned}
N(r, \Phi) \leq & \bar{N}_{(2}\left(r, \frac{1}{f}\right)+\bar{N}_{(2}\left(r, \frac{1}{g}\right)+N_{0}\left(r, \frac{1}{f^{\prime}}\right) \\
& +N_{0}\left(r, \frac{1}{g^{\prime}}\right)+\bar{N}_{L}\left(r, \frac{1}{f-1}\right)+\bar{N}_{L}\left(r, \frac{1}{g-1}\right),
\end{aligned}
$$

where $N_{0}\left(r, 1 / f^{\prime}\right)$ is the counting function which only counts those points such that $f^{\prime}=0$, but $f(f-1) \neq 0$. By the second fundamental theorem, we have

$$
\begin{aligned}
T(r, g) \leq & \bar{N}\left(r, \frac{1}{g}\right)+\bar{N}\left(r, \frac{1}{g-1}\right) \\
& -N_{0}\left(r, \frac{1}{g^{\prime}}\right)+S(r, g),
\end{aligned}
$$

since

$$
\begin{aligned}
\bar{N}\left(r, \frac{1}{g-1}\right)= & N_{11}\left(r, \frac{1}{g-1}\right)+\bar{N}_{(2}\left(r, \frac{1}{f-1}\right) \\
& +\bar{N}_{g>1}\left(r, \frac{1}{f-1}\right) .
\end{aligned}
$$

Thus, we deduce from (11)-(14) that

$$
\begin{aligned}
T(r, g) \leq & \bar{N}\left(r, \frac{1}{g}\right)+\bar{N}_{(2}\left(r, \frac{1}{f}\right) \\
& +\bar{N}_{(2}\left(r, \frac{1}{g}\right)+N_{0}\left(r, \frac{1}{f^{\prime}}\right) \\
& +\bar{N}_{(2}\left(r, \frac{1}{f-1}\right)+\bar{N}_{L}\left(r, \frac{1}{f-1}\right) \\
& +\bar{N}_{L}\left(r, \frac{1}{g-1}\right)+\bar{N}_{g>1}\left(r, \frac{1}{f-1}\right) \\
& +S(r, f)+S(r, g) .
\end{aligned}
$$

From the definition of $N_{0}\left(r, 1 / f^{\prime}\right)$, we see that

$$
\begin{aligned}
N_{0}\left(r, \frac{1}{f^{\prime}}\right) & +\bar{N}_{(2}\left(r, \frac{1}{f-1}\right)+N_{(2}\left(r, \frac{1}{f}\right) \\
& -\bar{N}_{(2}\left(r, \frac{1}{f}\right) \leq N\left(r, \frac{1}{f^{\prime}}\right) .
\end{aligned}
$$

The above inequality and Lemma 12 give

$$
\begin{aligned}
& N_{0}\left(r, \frac{1}{f^{\prime}}\right)+\bar{N}_{(2}\left(r, \frac{1}{f-1}\right) \\
& \quad \leq N\left(r, \frac{1}{f^{\prime}}\right)-N_{(2}\left(r, \frac{1}{f}\right)+\bar{N}_{(2}\left(r, \frac{1}{f}\right) \\
& \quad \leq N\left(r, \frac{1}{f}\right)-N_{(2}\left(r, \frac{1}{f}\right)+\bar{N}_{(2}\left(r, \frac{1}{f}\right)+S(r, f) \\
& \quad \leq \bar{N}\left(r, \frac{1}{f}\right)+S(r, f) .
\end{aligned}
$$


Substituting (17) in (15), we get

$$
\begin{aligned}
T(r, g) \leq & \bar{N}\left(r, \frac{1}{g}\right)+\bar{N}_{(2}\left(r, \frac{1}{f}\right)+\bar{N}_{(2}\left(r, \frac{1}{g}\right) \\
& +\bar{N}\left(r, \frac{1}{f}\right)+\bar{N}_{L}\left(r, \frac{1}{f-1}\right)+\bar{N}_{L}\left(r, \frac{1}{g-1}\right) \\
& +\bar{N}_{g>1}\left(r, \frac{1}{f-1}\right)+S(r, f)+S(r, g) \\
\leq & N_{2}\left(r, \frac{1}{g}\right)+\bar{N}_{(2}\left(r, \frac{1}{f}\right)+\bar{N}\left(r, \frac{1}{f}\right) \\
& +\bar{N}_{L}\left(r, \frac{1}{f-1}\right)+\bar{N}_{L}\left(r, \frac{1}{g-1}\right) \\
& +\bar{N}_{g>1}\left(r, \frac{1}{f-1}\right)+S(r, f)+S(r, g),
\end{aligned}
$$

since

$$
\begin{aligned}
\bar{N}_{L}\left(r, \frac{1}{f-1}\right) & \leq N\left(r, \frac{1}{f-1}\right)-\bar{N}\left(r, \frac{1}{f-1}\right) \\
& \leq N\left(r, \frac{f}{f^{\prime}}\right) \leq N\left(r, \frac{f^{\prime}}{f}\right)+S(r, f) \\
& \leq \bar{N}\left(r, \frac{1}{f}\right)+S(r, f) .
\end{aligned}
$$

Similarly,

$$
\bar{N}_{L}\left(r, \frac{1}{g-1}\right) \leq \bar{N}\left(r, \frac{1}{g}\right)+S(r, g) .
$$

Combining the above inequalities, Lemma 13, and (18), we obtain

$$
\begin{aligned}
T(r, g) \leq & N_{2}\left(r, \frac{1}{g}\right)+N_{2}\left(r, \frac{1}{f}\right)+\bar{N}\left(r, \frac{1}{f}\right)+2 \bar{N}\left(r, \frac{1}{g}\right) \\
& -N_{0}\left(r, \frac{1}{g^{\prime}}\right)+S(r, f)+S(r, g) \\
\leq & N_{2}\left(r, \frac{1}{g}\right)+N_{2}\left(r, \frac{1}{f}\right)+\bar{N}\left(r, \frac{1}{f}\right) \\
& +2 \bar{N}\left(r, \frac{1}{g}\right)+S(r, f)+S(r, g) .
\end{aligned}
$$

Thus, we obtain (i).

If $\Phi(z) \equiv 0$, then by (10), we have

$$
\frac{f^{\prime \prime}}{f^{\prime}}-\frac{2 f^{\prime}}{f-1} \equiv \frac{g^{\prime \prime}}{g^{\prime}}-\frac{2 g^{\prime}}{g-1} .
$$

By integrating two sides of the above equality, we obtain

$$
f \equiv \frac{A g+B}{C g+D}
$$

where $A, B, C$, and $D$ are finite complex numbers satisfying $A D \neq B C$. This proves the lemma.
Lemma 15 (see [14]). Let $f(z)$ be a nonconstant meromorphic function, $s, k$ be two positive integers; then

$$
\begin{aligned}
N_{s}\left(r, \frac{1}{f^{(k)}}\right) \leq & T(r, f)^{(k)}-T(r, f) \\
& +N_{s+k}\left(r, \frac{1}{f}\right)+S(r, f), \\
N_{s}\left(r, \frac{1}{f^{(k)}}\right) \leq & k \bar{N}(r, f) \\
& +N_{s+k}\left(r, \frac{1}{f}\right)+S(r, f) .
\end{aligned}
$$

Clearly, $\bar{N}\left(r, 1 / f^{(k)}\right)=N_{1}\left(r, 1 / f^{(k)}\right)$.

Lemma 16 (see $[15])$. Let $a_{0}(z), a_{1}(z), \ldots, a_{n}(z), b(z)$ be polynomials such that $a_{0}(z) a_{n}(z) \neq \equiv$; let $c_{j}$ be constants and

$$
\operatorname{deg}\left(\sum_{\operatorname{deg} a_{j}=d} a_{j}\right)=d
$$

where $d=\max _{0 \leq j \leq n}\left\{\operatorname{deg} a_{j}\right\}$. If $f(z)$ is a transcendental meromorphic solution of

$$
\sum_{j=0}^{n} a_{j}(z) f\left(z+c_{j}\right)=b(z),
$$

then $\rho(f) \geq 1$.

\section{Proof of Theorems}

3.1. Proof of Theorem 4. Let $G(z)=f(z)^{n}(f(z+c)-1) \Delta_{c} f$. Since $f$ is a transcendental entire function of finite order, from Lemma 7, we have

$$
\begin{aligned}
(n & +2) T(r,(r, f(z))) \\
& \leq T\left(r, f(z)^{n+1}(f(z+c)-1)\right)+S(r, f) \\
& \leq m\left(r, f(z)^{n+1}(f(z+c)-1)\right)+S(r, f) \\
& \leq m\left(r, \frac{f(z)^{n+1}(f(z+c)-1)}{G}\right)+m(r, G)+S(r, f) \\
& \leq T(r, G)+S(r, f) .
\end{aligned}
$$

By the second main theorem, we deduce that

$$
\begin{aligned}
T(r, G) \leq & \bar{N}(r, G)+\bar{N}\left(r, \frac{1}{G}\right)+\bar{N}\left(r, \frac{1}{G-a}\right)+S(r, G) \\
\leq & \bar{N}\left(r, \frac{1}{G-a}\right)+\bar{N}\left(r, \frac{1}{f}\right)+\bar{N}\left(r, \frac{1}{f(z+c)-1}\right) \\
& +\bar{N}\left(r, \frac{1}{\Delta_{c} f}\right)+S(r, f)
\end{aligned}
$$




$$
\begin{aligned}
\leq & \bar{N}\left(r, \frac{1}{G-a}\right)+\bar{N}\left(r, \frac{1}{f}\right)+T(r, f(z+c)-1) \\
& +T\left(r, \Delta_{c} f\right)+S(r, f) \\
\leq & \bar{N}\left(r, \frac{1}{G-a}\right)+\bar{N}\left(r, \frac{1}{f}\right)+T(r, f(z+c)-1) \\
& +m\left(r, \frac{\Delta_{c} f}{f} \cdot f\right)+S(r, f) \\
\leq & \bar{N}\left(r, \frac{1}{G-a}\right)+\bar{N}\left(r, \frac{1}{f}\right)+T(r, f(z+c)-1) \\
& +m\left(r, \frac{\Delta_{c} f}{f}\right)+m(r, f)+S(r, f) \\
\leq & \bar{N}\left(r, \frac{1}{G-a}\right)+3 T(r, f)+S(r, f) .
\end{aligned}
$$

According to (27) and (28), we have

$$
(n-1) T(r, f) \leq \bar{N}\left(r, \frac{1}{G-a}\right)+S(r, f) .
$$

Noting that $n \geq 2$, we get that $G-a$ has infinitely many zeros.

This completes the proof of Theorem 4 .

3.2. Proof of Theorem 5. Since $\left[f(z)^{n} \Delta_{c} f\right]^{(k)}$ and $\left[g(z)^{n} \Delta_{c} g\right]^{(k)}$ share $1 \mathrm{CM}$, we have

$$
\frac{\left[f(z)^{n} \Delta_{c} f\right]^{(k)}-1}{\left[g(z)^{n} \Delta_{c} g\right]^{(k)}-1}=e^{h(z)}
$$

where $h(z)$ is a polynomial. Set $F=f(z)^{n} \Delta_{c} f, G=$ $g(z)^{n} \Delta_{c} g$,

$$
\begin{array}{r}
F_{1}=F^{(k)}, \quad F_{2}=-e^{h(z)} G^{(k)}, \quad F_{3}=e^{h(z),} \\
\text { then } F_{1}+F_{2}+F_{3}=1, \\
T(r)=\max _{1 \leq j \leq 3} T\left(r, F_{j}\right), \quad S(r)=o(T(r)) .
\end{array}
$$

Next, we will prove that $F_{1}, F_{2}$, and $F_{3}$ are linearly dependent and either $F_{2}$ or $F_{3}$ is a constant.

Now, we suppose that neither $F_{2}$ nor $F_{3}$ is a constant and $F_{1}, F_{2}$, and $F_{3}$ are linearly independent; then by Lemma 9 , we have

$$
T\left(r, F_{1}\right) \leq \sum_{j=1}^{3} N_{2}\left(r, \frac{1}{F_{j}}\right)+\sum_{j=1}^{3} \bar{N}\left(r, F_{j}\right)+o(T(r))
$$

Since $F_{j}(j=1,2,3)$ are entire functions, by the above inequality, we get

$$
T\left(r, F_{1}\right) \leq N_{2}\left(r, \frac{1}{F^{(k)}}\right)+N_{2}\left(r, \frac{1}{G^{(k)}}\right)+o(T(r)) .
$$

From (33) and the first main theorem, we have

$$
\begin{aligned}
T\left(r, \frac{1}{F^{(k)}}\right)= & T\left(r, F^{(k)}\right)+O(1)=T\left(r, F_{1}\right)+O(1) \\
\leq & N_{2}\left(r, \frac{1}{F^{(k)}}\right)+N_{2}\left(r, \frac{1}{G^{(k)}}\right)+o(T(r)) \\
\leq & N\left(r, \frac{1}{F^{(k)}}\right) \\
& -\left[N_{(3}\left(r, \frac{1}{F^{(k)}}\right)-2 \bar{N}_{(3}\left(r, \frac{1}{F^{(k)}}\right)\right] \\
& +N\left(r, \frac{1}{G^{(k)}}\right) \\
& -\left[N_{(3}\left(r, \frac{1}{G^{(k)}}\right)-2 \bar{N}_{(3}\left(r, \frac{1}{G^{(k)}}\right)\right] \\
& +o(T(r)) .
\end{aligned}
$$

Assuming that $z_{0}$ is zero of $f(z)$ (or $g(z)$ ) with multiplicity $p$, if $z_{0}$ is zero of $f(z+c)$ (or $g(z+c)$ ) with multiplicity $q(\geq 1)$, let $m=\min \{p, q\}$, then $z_{0}$ is a zero of $F^{(k)}$ (or $\left.G^{(k)}\right)$ with multiplicity $n p+m-k \geq n p-k \geq 3$, and if $z_{0}$ is not zero of $f(z+c)$ (or $g(z+c))$, then $z_{0}$ is a zero of $F^{(k)}\left(\right.$ or $\left.G^{(k)}\right)$ with multiplicity $n p-k \geq 3$. Therefore, we get that

$$
N_{(3}\left(r, \frac{1}{F^{(k)}}\right)-2 \bar{N}_{(3}\left(r, \frac{1}{F^{(k)}}\right) \geq(n-k-2) N\left(r, \frac{1}{f}\right),
$$

$$
N_{(3}\left(r, \frac{1}{G^{(k)}}\right)-2 \bar{N}_{(3}\left(r, \frac{1}{G^{(k)}}\right) \geq(n-k-2) N\left(r, \frac{1}{g}\right),
$$

since

$$
\begin{aligned}
n m\left(r, \frac{1}{f}\right)= & m\left(r, \frac{1}{f^{n}}\right)=m\left(r, \frac{\Delta_{c} f}{F}\right) \\
\leq & m\left(r, \frac{1}{F}\right)+m\left(r, \frac{\Delta_{c} f}{f} \cdot f\right) \\
\leq & m\left(r, \frac{F^{(k)}}{F} \cdot \frac{1}{F^{(k)}}\right)+m\left(r, \frac{\Delta_{c} f}{f}\right) \\
& +m(r, f)+S(r, f) \\
\leq & m\left(r, \frac{1}{F^{(k)}}\right)+T(r, f)+S(r, f) \\
= & T\left(r, \frac{1}{F^{(k)}}\right)-N\left(r, \frac{1}{F^{(k)}}\right)+T(r, f)+S(r, f) .
\end{aligned}
$$

Therefore, from (34), (35), (36), (37), and Lemma 12,

$$
\begin{aligned}
(n-1) T(r, f) \leq & (k+2) N\left(r, \frac{1}{f}\right)+(k+2) N\left(r, \frac{1}{g}\right) \\
& +T(r, g)+o(T(r)) .
\end{aligned}
$$


On the other hand, from (30), we have $G^{(k)}+e^{-h}-e^{-h} F^{(k)}=1$. Obviously, according to our assumptions, neither $e^{-h}$ nor $e^{-h} F^{(k)}$ is a constant and $F_{1}, F_{2}$, and $F_{3}$ are linearly independent. Similarly, we have

$$
\begin{aligned}
(n-1) T(r, g) \leq & (k+2) N\left(r, \frac{1}{g}\right)+(k+2) N\left(r, \frac{1}{f}\right) \\
& +T(r, f)+o(T(r)) .
\end{aligned}
$$

From (38) and (39), we obtain that

$$
[n-2 k-6](T(r, f)+T(r, g)) \leq o(T(r)),
$$

which is a contradiction to $n \geq 2 k+7$.

Therefore, $F_{1}, F_{2}$, and $F_{3}$ are linearly dependent, and there exist constants $C_{1}, C_{2}, C_{3}$ which are not all equal to zero such that

$$
C_{1} F_{1}+C_{2} F_{2}+C_{3} F_{3}=0 .
$$

Suppose that $C_{1}=0$; we have $C_{2} F_{2}+C_{3} F_{3}=0$. If $C_{2} \neq 0$, we get $F_{2}=-\left(C_{3} / C_{2}\right) F_{3}$; that is, $G^{(k)}=C_{3} / C_{2}$; thus $g(z)$ is a polynomial; it is impossible. Similarly, if $C_{2}=0$, we also deduce a contradiction.

Suppose that $C_{1} \neq 0$, from (41); we know that $\left(C_{2}, C_{3}\right) \neq(0,0)$. If $C_{2} \neq 0$, from (41), we have

$$
\left(1-\frac{C_{2}}{C_{1}}\right) F_{2}+\left(1-\frac{C_{3}}{C_{1}}\right) F_{3}=1
$$

and $C_{1} \neq C_{2}, C_{1} \neq C_{3}$. That is,

$$
\left(1-\frac{C_{2}}{C_{1}}\right) G^{(k)}+\frac{1}{e^{h}}=1-\frac{C_{3}}{C_{1}} .
$$

From Lemma 11, we have

$$
\begin{aligned}
T\left(r, G^{(k)}\right) \leq & \bar{N}\left(r, \frac{1}{G^{(k)}}\right)+\bar{N}\left(r, G^{(k)}\right)+\bar{N}\left(r, e^{h}\right)+S(r, g) \\
= & \bar{N}\left(r, \frac{1}{G^{(k)}}\right)+S(r, g) \leq N\left(r, \frac{1}{G^{(k)}}\right) \\
& -\left[N_{(2}\left(r, \frac{1}{G^{(k)}}\right)-\bar{N}_{(2}\left(r, \frac{1}{G^{(k)}}\right)\right]+S(r, g) .
\end{aligned}
$$

By the similar argument in (37), we have

$$
\begin{aligned}
n m\left(r, \frac{1}{g}\right) \leq & T\left(r, \frac{1}{G^{(k)}}\right)-N\left(r, \frac{1}{G^{(k)}}\right) \\
& +T(r, g)+S(r, g) .
\end{aligned}
$$

From $n \geq 2 k+7>k+2$, if $z_{0}$ is zero of $g(z)$ with multiplicity $p$, then $z_{0}$ is a zero of $G^{(k)}$ with multiplicity $n p-k \geq 2$, and we get

$$
N_{(2}\left(r, \frac{1}{G^{(k)}}\right)-\bar{N}_{(2}\left(r, \frac{1}{G^{(k)}}\right) \geq(n-k-1) N\left(r, \frac{1}{g}\right) .
$$

According to (44), (45), and (46), we have

$$
(n-1) T(r, g) \leq(k+1) N\left(r, \frac{1}{g}\right)+S(r, g),
$$

which is a contradiction to $n \geq 2 k+7$.

Therefore, $C_{2}=0, C_{3} \neq 0$, which gives $\left(1-C_{1} / C_{3}\right) F_{1}+F_{2}=$ 1. Similarly, we derive a contradiction by calculation.

Hence, we deduce that either $F_{2}$ or $F_{3}$ is a constant.

Suppose $F_{2}=c \neq 1$; from $F_{1}+F_{2}+F_{3}=1$, we have $F^{(k)}+e^{h}=1-c$; in the same manner as above, we get a contradiction. Therefore, $c=1$; that is, $F_{2}=1$. Suppose $F_{3}=c \neq 1$; similarly as above, we get $c=1$; that is, $F_{3}=1$.

Therefore, we conclude that $F_{2}=1$ or $F_{3}=1$.

If $F_{2}=1$, since $F_{1}+F_{2}+F_{3}=1$, we have $F_{1}=-F_{3}=-e^{h(z)}$. That is

$$
\left[f^{n} \Delta_{c} f\right]^{(k)} \cdot\left[g^{n} \Delta_{c} g\right]^{(k)} \equiv 1 .
$$

Since $n \geq 2 k+7$ and $f$ and $g$ are transcendental entire functions with hyperorder less than one, we get that $f$ and $g$ have no zeros. Thus,

$$
f(z)=e^{a(z)}, \quad g(z)=e^{b(z)},
$$

where $a(z), b(z)$ are nonzero polynomials.

Substitute (49) into (48); we have

$$
\left[e^{n a(z)}\left(e^{a(z+c)}-e^{a(z)}\right)\right]^{(k)} \cdot\left[e^{n b(z)}\left(e^{b(z+c)}-e^{b(z)}\right)\right]^{(k)} \equiv 1 .
$$

Let $n a(z)+a(z+c)=A_{1}, n a(z)+a(z)=A_{2}, n b(z)+b(z+c)=$ $B_{1}$, and $n b(z)+b(z)=B_{2}$. If $k=1$, we have

$$
\left(A_{1}^{\prime} e^{A_{1}}-A_{2}^{\prime} e^{A_{2}}\right) \cdot\left(B_{1}^{\prime} e^{B_{1}}-B_{2}^{\prime} e^{B_{2}}\right) \equiv 1 .
$$

From (51), we know that $A_{1}^{\prime} e^{A_{1}}-A_{2}^{\prime} e^{A_{2}}=e^{A_{2}}\left(A_{1}^{\prime} e^{A_{1}-A_{2}}-\right.$ $\left.A_{2}^{\prime}\right) \neq 0$; If $A_{1}^{\prime} \neq 0$, then we have $A_{2}^{\prime}=0$; thus, $A_{2}$ must be a constant. By Lemma 16, we have $\rho(a(z)) \geq 1$; thus, $\rho_{2}(f) \geq 1$, which is a contradiction. If $A_{1}^{\prime}=0$, then $A_{1}$ must be a constant; similarly, we also deduce a contradiction.

If $k=2$, by calculation, we have

$$
\begin{aligned}
A_{1}^{\prime \prime} e^{A_{1}} & +\left(A_{1}^{\prime}\right)^{2} e^{A_{1}}-A_{2}^{\prime \prime} e^{A_{2}}-\left(A_{2}^{\prime}\right)^{2} e^{A_{2}} \\
& =e^{A_{2}}\left[e^{A_{1}-A_{2}}\left(A_{1}^{\prime \prime}+\left(A_{1}^{\prime}\right)^{2}\right)-\left(A_{2}^{\prime \prime}+\left(A_{2}^{\prime}\right)^{2}\right)\right] \neq 0 .
\end{aligned}
$$

If $A_{1}^{\prime \prime}+\left(A_{1}^{\prime}\right)^{2} \neq 0$, then $A_{2}^{\prime \prime}+\left(A_{2}^{\prime}\right)^{2}=0$. If $A_{2}$ is transcendental entire, then we have

$$
m\left(r, A_{2}^{\prime}\right)=m\left(\frac{A_{2}^{\prime \prime}}{A_{2}^{\prime}}\right)=S\left(r, A_{2}^{\prime}\right),
$$

which is a contradiction to $A_{2}^{\prime}$ being transcendental entire. If $A_{2}$ is a polynomial, from Lemma 16, which induces that $\rho_{2}(f) \geq 1$, we get a contradiction. If $A_{1}^{\prime \prime}+\left(A_{1}^{\prime}\right)^{2}=0$, similar as above, we get a contradiction. For $k \geq 3$, using the similar 
Method as above, we also deduce a contradiction. Therefore, There are not transcendental entire functions $f(z)$ and $g(z)$ satisfying (48).

If $F_{3}=1$, that is, $e^{h(z)}=1$, from (30), we get

$$
\left[f^{n} \Delta_{c} f\right]^{(k)} \equiv\left[g^{n} \Delta_{c} g\right]^{(k)} \text {. }
$$

From (54), we have

$$
f^{n} \Delta_{c} f \equiv g^{n} \Delta_{c} g+p(z),
$$

where $p(z)$ is a polynomial of degree at most $k-1$. Suppose $p(z) \neq 0$; then we get

$$
\frac{f^{n} \Delta_{c} f}{p(z)}=\frac{g^{n} \Delta_{c} g}{p(z)}+1 .
$$

Therefore, from the second main theorem, we have

$$
\begin{aligned}
(n+1) T(r, f) \leq & T\left(\frac{f^{n} \Delta_{c} f}{p(z)}\right)+S(r, f) \\
\leq & \bar{N}\left(\frac{f^{n} \Delta_{c} f}{p(z)}\right)+\bar{N}\left(\frac{p(z)}{f^{n} \Delta_{c} f}\right) \\
& +\bar{N}\left(\frac{p(z)}{g^{n} \Delta_{c} g}\right)+S(r, f) \\
\leq & \bar{N}\left(\frac{1}{f}\right)+\bar{N}\left(\frac{1}{\Delta_{c} f}\right)+\bar{N}\left(\frac{1}{g}\right) \\
& +\bar{N}\left(\frac{1}{\Delta_{c} g}\right)+S(r, f) \\
\leq & 2 T(r, f)+2 T(r, g)+S(r, f) .
\end{aligned}
$$

Similarly, we have

$$
(n+1) T(r, g) \leq 2 T(r, f)+2 T(r, g)+S(r, f) .
$$

Therefore,

$$
\begin{aligned}
& (n+1)[T(r, f)+T(r, g)] \\
& \quad \leq 4[T(r, f)+T(r, g)]+S(r, f)+S(r, g),
\end{aligned}
$$

which is a contradiction to $n \geq 2 k+7$. Thus, $p(z) \equiv 0$, which implies that

$$
f^{n} \Delta_{c} f \equiv g^{n} \Delta_{c} g
$$

Let $f / g=h$; if $h$ is not a constant, then by (60), we have

$$
h^{n+1} \equiv \frac{f}{\Delta_{c} f} \cdot \frac{\Delta_{c} g}{g} .
$$

Thus,

$$
\begin{aligned}
(n+1) T(r, h) \leq & T\left(r, \frac{\Delta_{c} f}{f}\right)+T\left(r, \frac{\Delta_{c} g}{g}\right)+O(1) \\
\leq & N\left(r, \frac{\Delta_{c} f}{f}\right)+N\left(r, \frac{\Delta_{c} g}{g}\right) \\
& +S(r, f)+S(r, g) \\
\leq & T(r, f)+T(r, g)+S(r, f)+S(r, g) .
\end{aligned}
$$

Combining $T(r, h)=T(r, f / g)=T(r, f)+T(r, g)+O(1)$, we obtain $n(T(r, f)+T(r, g)) \leq S(r, f)+S(r, g)$, which is impossible.

Therefore, $h$ is a constant; then substituting $f=g h$ into (60), we have $h^{n+1} \equiv 1$. Hence $f(z)=\operatorname{tg}(z)$, where $t$ is a constant and $t^{n+1}=1$.

The proof of Theorem 5 is complete.

\subsection{Proof of Theorem 6. Let}

$$
\begin{array}{cc}
F(z)=\left[f(z)^{n} \Delta_{c} f\right]^{(k)}, & G(z)=\left[g(z)^{n} \Delta_{c} g\right]^{(k)}, \\
F_{1}(z)=f(z)^{n} \Delta_{c} f, & G_{1}(z)=g(z)^{n} \Delta_{c} g .
\end{array}
$$

Then $F(z)$ and $G(z)$ share $1 \mathrm{IM}$, and $F_{1}^{(k)}=F, G_{1}^{(k)}=G$. By Lemma 10, we have

$$
\begin{aligned}
& n T(r, f)+S(r, f) \leq T\left(r, F_{1}\right) \leq(n+1) T(r, f)+S(r, f), \\
& n T(r, g)+S(r, g) \leq T\left(r, G_{1}\right) \leq(n+1) T(r, g)+S(r, g) .
\end{aligned}
$$

Since $f$ is transcendental entire, by the definition of $F$, we have

$$
\begin{aligned}
N_{2}\left(r, \frac{1}{F}\right) & =\bar{N}\left(r, \frac{1}{F}\right)+\bar{N}_{(2}\left(r, \frac{1}{F}\right) \\
& =N\left(r, \frac{1}{F}\right)-\left[N_{(3}\left(r, \frac{1}{F}\right)-2 \bar{N}_{(3}\left(r, \frac{1}{F}\right)\right] .
\end{aligned}
$$

Using the argument in (35), we have

$$
N_{(3}\left(r, \frac{1}{F}\right)-2 \bar{N}_{(3}\left(r, \frac{1}{F}\right) \geq(n-k-2) N\left(r, \frac{1}{f}\right) .
$$

It follows from Lemma 12 and (66), (67), we have

$$
\begin{aligned}
N_{2}\left(r, \frac{1}{F}\right) \leq & N\left(r, \frac{1}{F}\right)-(n-k-2) N\left(r, \frac{1}{f}\right) \\
\leq & N\left(r, \frac{1}{f^{n} \Delta_{c} f}\right)-(n-k-2) N\left(r, \frac{1}{f}\right) \\
& +S(r, f) \leq n N\left(r, \frac{1}{f}\right)+N\left(r, \frac{1}{\Delta_{c} f}\right) \\
& -(n-k-2) N\left(r, \frac{1}{f}\right)+S(r, f) \\
\leq & (k+3) T(r, f)+S(r, f) .
\end{aligned}
$$

From Lemma 15, we have

$$
\begin{aligned}
\bar{N}\left(r, \frac{1}{F}\right) & \leq N_{k+1}\left(r, \frac{1}{f^{n} \Delta_{c} f}\right)+S(r, f) \\
& \leq(k+1) \bar{N}\left(r, \frac{1}{f}\right)+N\left(r, \frac{1}{\Delta_{c} f}\right)+S(r, f) \\
& \leq(k+2) T(r, f)+S(r, f) .
\end{aligned}
$$


Similarly,

$$
\begin{aligned}
& N_{2}\left(r, \frac{1}{G}\right) \leq(k+3) T(r, g)+S(r, g), \\
& \bar{N}\left(r, \frac{1}{G}\right) \leq(k+2) T(r, g)+S(r, f) .
\end{aligned}
$$

By Lemma 14, one of the following cases holds:

(i) $T(r, G) \leq N_{2}(r, 1 / G)+N_{2}(r, 1 / F)+\bar{N}(r, 1 / F)+$ $2 \bar{N}(r, 1 / G)+S(r, F)+S(r, G)$, the same inequality holding for $T(r, F)$;

(ii) $F \equiv(A G+B) /(C G+D)$.

For case (i), we have

$$
\begin{aligned}
T(r, G) \leq & N_{2}\left(r, \frac{1}{G}\right)+N_{2}\left(r, \frac{1}{F}\right)+\bar{N}\left(r, \frac{1}{F}\right) \\
& +2 \bar{N}\left(r, \frac{1}{G}\right)+S(r, F)+S(r, G), \\
T(r, F) \leq & N_{2}\left(r, \frac{1}{F}\right)+N_{2}\left(r, \frac{1}{G}\right)+\bar{N}\left(r, \frac{1}{G}\right) \\
& +2 \bar{N}\left(r, \frac{1}{F}\right)+S(r, F)+S(r, G) .
\end{aligned}
$$

Therefore, we get

$$
\begin{aligned}
T(r, F)+T(r, G) \leq & 2\left[N_{2}\left(r, \frac{1}{F}\right)+N_{2}\left(r, \frac{1}{G}\right)\right] \\
& +3\left[\bar{N}\left(r, \frac{1}{G}\right)+\bar{N}\left(r, \frac{1}{F}\right)\right] \\
& +S(r, F)+S(r, G) .
\end{aligned}
$$

By (64) and Lemma 15, we have

$$
\begin{aligned}
n T(r, f) \leq & T\left(r, F_{1}\right)+S(r, f) \leq T(r, F)-N_{2}\left(r, \frac{1}{F}\right) \\
& +N_{k+2}\left(r, \frac{1}{F_{1}}\right)+S(r, f) \\
\leq & T(r, F)-N_{2}\left(r, \frac{1}{F}\right)+(k+2) \bar{N}\left(r, \frac{1}{f}\right) \\
& +N\left(r, \frac{1}{\Delta_{c} f}\right)+S(r, f) \\
\leq & T(r, F)-N_{2}\left(r, \frac{1}{F}\right) \\
& +(k+3) T(r, f)+S(r, f) .
\end{aligned}
$$

Similarly,

$n T(r, g) \leq T(r, G)-N_{2}\left(r, \frac{1}{G}\right)+(k+3) T(r, g)+S(r, g)$.

By (70), (72), (73), and (74), we obtain

$$
(n-5 k-12)\{T(r, f)+T(r, g)\} \leq S(r, f)+S(r, g),
$$

which is a contradiction since $n \geq 5 k+13$.
For case (ii), we have

$$
F \equiv \frac{A G+B}{C G+D}
$$

where $A, B, C$, and $D$ are finite complex numbers satisfying $A D \neq B C$. Therefore, by the first fundamental theorem, $T(r, F)=T(r, G)+S(r, F)$.

Next, we consider three cases.

Case 1. $A C \neq 0$; from (76), we get

$$
F-\frac{A}{C}=\frac{B-A D / C}{C G+D} .
$$

By the second fundamental theorem and (69), we have

$$
\begin{aligned}
T(r, F) & \leq \bar{N}\left(r, \frac{1}{F-A / C}\right)+\bar{N}\left(r, \frac{1}{F}\right)+S(r, F) \\
& =\bar{N}(r, G)+(k+2) T(r, f)+S(r, F) \\
& \leq(k+2) T(r, f)+S(r, F) .
\end{aligned}
$$

From (73), we obtain $(n-2 k-5) T(r, f) \leq S(r, f)$, contradicting to $n \geq 5 k+13$.

Case 2. $A \neq 0$, and $C=0$. Then, $F \equiv A G+B / D$.

If $B \neq 0$, by the second fundamental theorem and (69), (70), we have

$$
\begin{aligned}
T(r, F) & \leq \bar{N}\left(r, \frac{1}{F-B / D}\right)+\bar{N}\left(r, \frac{1}{F}\right)+S(r, F) \\
& =\bar{N}\left(r, \frac{1}{G}\right)+\bar{N}\left(r, \frac{1}{F}\right)+S(r, F) \\
& \leq(k+2) T(r, f)+(k+2) T(r, g)+S(r, F) .
\end{aligned}
$$

Similarly,

$$
T(r, G) \leq(k+2) T(r, f)+(k+2) T(r, g)+S(r, G) .
$$

From (73), (74), (79), and (80), we get

$$
(n-3 k-7)[T(r, f)+T(r, g)] \leq S(r, F)+S(r, G),
$$

which is a contradiction to $n \geq 5 k+13$.

If $B=0$, then $F \equiv A G / D$. If $A / D=1$, then $F \equiv G$; that is, $\left[f^{n} \Delta_{c} f\right]^{(k)} \equiv\left[g^{n} \Delta_{c} g\right]^{(k)}$; using the argument in (54) and noting that $n \geq 5 k+13$, we obtain $f(z)=\operatorname{tg}(z)$, where $t$ is a constant and $t^{n+1}=1$. If $A / D \neq 1$, by the condition that $F$ and $G$ share $1 \mathrm{IM}$, then $F \neq 1$ and $G \neq 1$. we obtain then $F \neq 1$ and $F \neq A / D$. By the second fundamental theorem, we have

$$
T(r, F) \leq \bar{N}\left(\frac{1}{F-1}\right)+\bar{N}\left(\frac{1}{F-A / D}\right)+S(r, F) \leq S(r, F),
$$

which is impossible. 
Case 3. $A=0$, and $C \neq 0$. Then, $F \equiv B /(C G+D)$.

If $D \neq 0$, by the second fundamental theorem and (69), (70), we have

$$
\begin{aligned}
T(r, F) & \leq \bar{N}\left(r, \frac{1}{F-B / D}\right)+\bar{N}\left(r, \frac{1}{F}\right)+S(r, F) \\
& =\bar{N}\left(r, \frac{1}{G}\right)+\bar{N}\left(r, \frac{1}{F}\right)+S(r, F) \\
& \leq(k+2) T(r, f)+(k+2) T(r, g)+S(r, F) .
\end{aligned}
$$

Similarly,

$$
T(r, G) \leq(k+2) T(r, f)+(k+2) T(r, g)+S(r, G) .
$$

From (73), (74), (83), and (84), we get

$$
(n-3 k-7)[T(r, f)+T(r, g)] \leq S(r, F)+S(r, G),
$$

which is a contradiction to $n \geq 5 k+13$.

If $D=0$, then $F \equiv B / C G$. If $B / C=1$, then $F \cdot G \equiv 1$; using the argument in (48) in Theorem 5 and noting that $n \geq 5 k+$ 13 , we get a contradiction. If $B / C \neq 1$, by the condition that $F$ and $G$ share 1 IM, we obtain $F \neq 1$ and $F \neq B / C$. By the second fundamental theorem, we have

$$
T(r, F) \leq \bar{N}\left(\frac{1}{F-1}\right)+\bar{N}\left(\frac{1}{F-B / C}\right)+S(r, F) \leq S(r, F),
$$

which is impossible.

The proof of Theorem 6 is complete.

\section{Acknowledgments}

This work is supported by Youth Foundation of Chongqing Normal University (12XWQ17), partially by Chongqing City Board of Education Science, and Technology Project (KJ130632), and partially by the fund of Chongqing Normal University (13XLB006).

\section{References}

[1] W. K. Hayman, Meromorphic Functions, Clarendon Press, Oxford, UK, 1964.

[2] L. Yang, Value Distribution Theory, Springer, Berlin, Germany, 1993.

[3] C.-C. Yang and H.-X. Yi, Uniqueness Theory of Meromorphic Functions, Kluwer Academic, Dordrecht, The Netherlands, 2003.

[4] I. Laine, Nevanlinna Theory and Complex Differential Equations, vol. 15 of de Gruyter Studies in Mathematics, Walter de Gruyter \& Co., Berlin, Germany, 1993.

[5] I. Lahiri, "Weighted sharing and uniqueness of meromorphic functions," Nagoya Mathematical Journal, vol. 161, pp. 193-206, 2001.

[6] X.-G. Qi, L.-Z. Yang, and K. Liu, "Uniqueness and periodicity of meromorphic functions concerning the difference operator," Computers \& Mathematics with Applications, vol. 60, no. 6, pp. 1739-1746, 2010.
[7] J.1. Zhang, Z. S. Gao, and S. Li, "Distribution of zeros and shared values of difference operators," Annales Polonici Mathematici, vol. 102, no. 3, pp. 213-221, 2011.

[8] Y.-M. Chiang and S.-J. Feng, "On the Nevanlinna characteristic of $f(z+\eta)$ and difference equations in the complex plane," Ramanujan Journal, vol. 16, no. 1, pp. 105-129, 2008.

[9] R. G. Halburd and R. J. Korhonen, "Nevanlinna theory for the difference operator," Annales Academioe Scientiarum Fennicoe. Mathematica, vol. 31, no. 2, pp. 463-478, 2006.

[10] P. Li and C.-C. Yang, "Some further results on the unique range sets of meromorphic functions," Kodai Mathematical Journal, vol. 18, no. 3, pp. 437-450, 1995.

[11] H.X. Yi, "Uniqueness of meromorphic functions and a question of C. C. Yang," Complex Variables. Theory and Application, vol. 14, no. 1-4, pp. 169-176, 1990.

[12] H. X. Yi and C. C. Yang, Uniqueness Theory of Meromorphic Functions, Science Press, Beijng, China, 1995.

[13] A. Banerjee, "Meromorphic functions sharing one value," International Journal of Mathematics and Mathematical Sciences, vol. 2005, no. 22, pp. 3587-3598, 2005.

[14] I. Lahiri and A. Sarkar, "Uniqueness of a meromorphic function and its derivative," Journal of Inequalities in Pure and Applied Mathematics, vol. 5, no. 1, article 20, 2004.

[15] K. Liu, X. L. Liu, and T. B. Cao, "Some results on zeros distributions anduniqueness of derivatives of difference polynomials," http://arxiv.org/abs/1107.0773v1. 


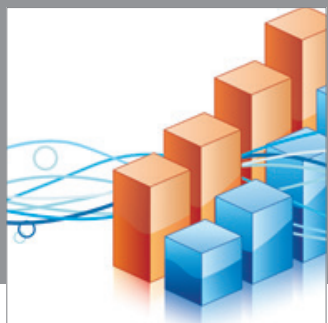

Advances in

Operations Research

mansans

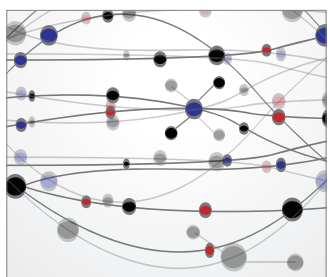

The Scientific World Journal
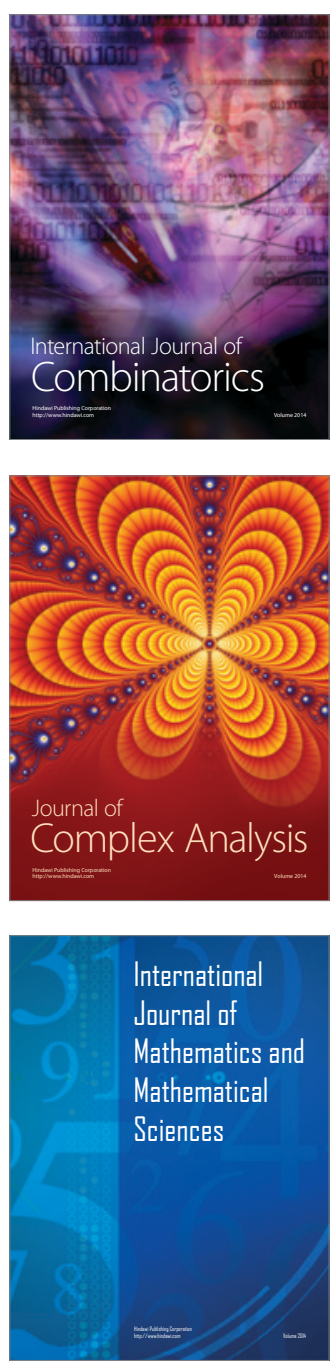
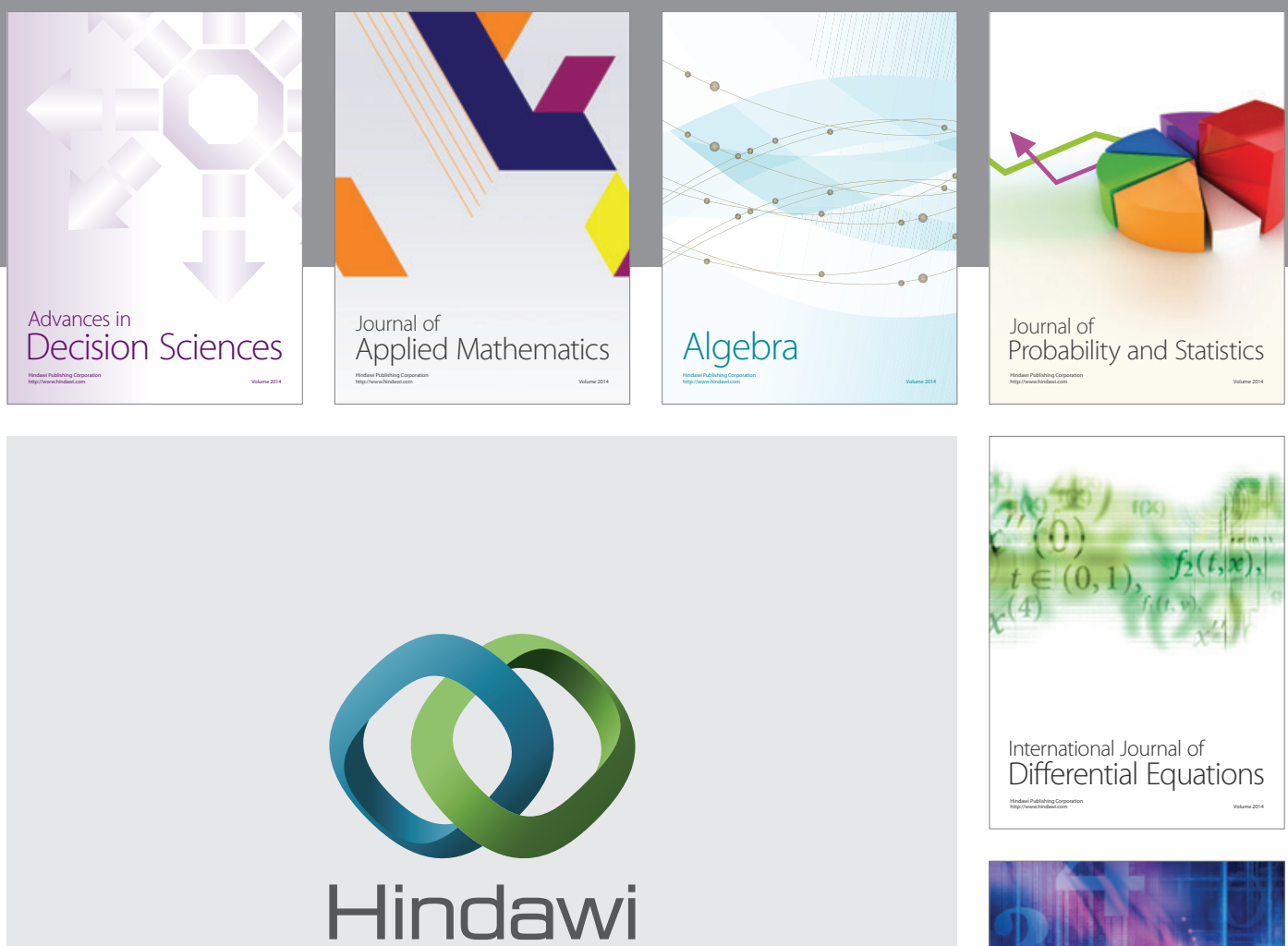

Submit your manuscripts at http://www.hindawi.com
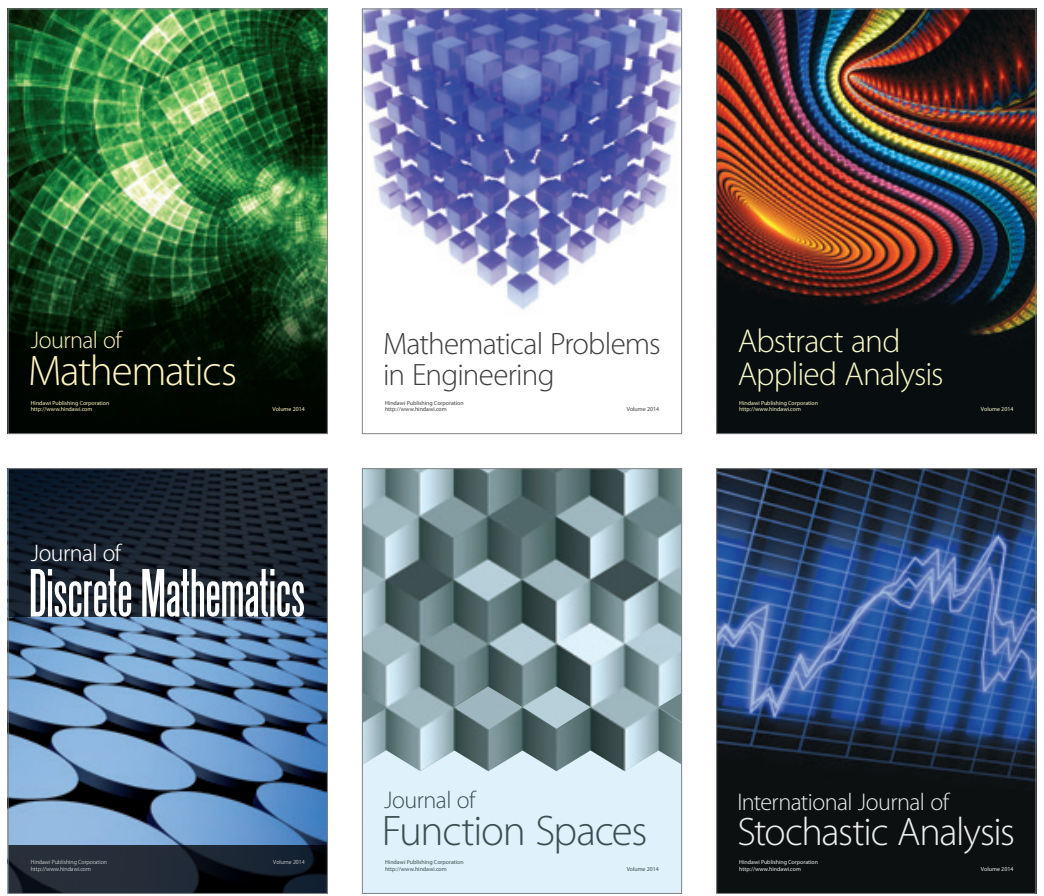

Journal of

Function Spaces

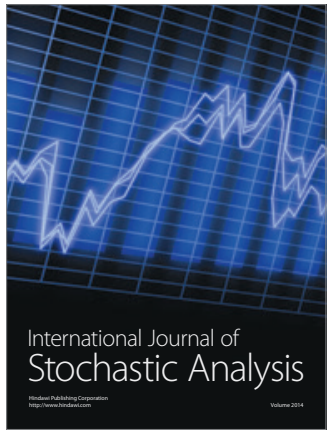

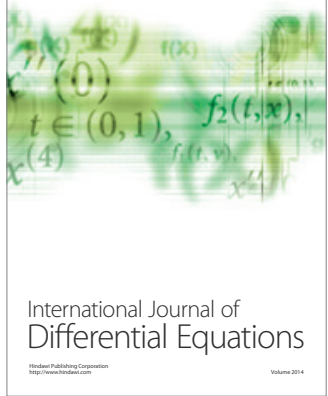
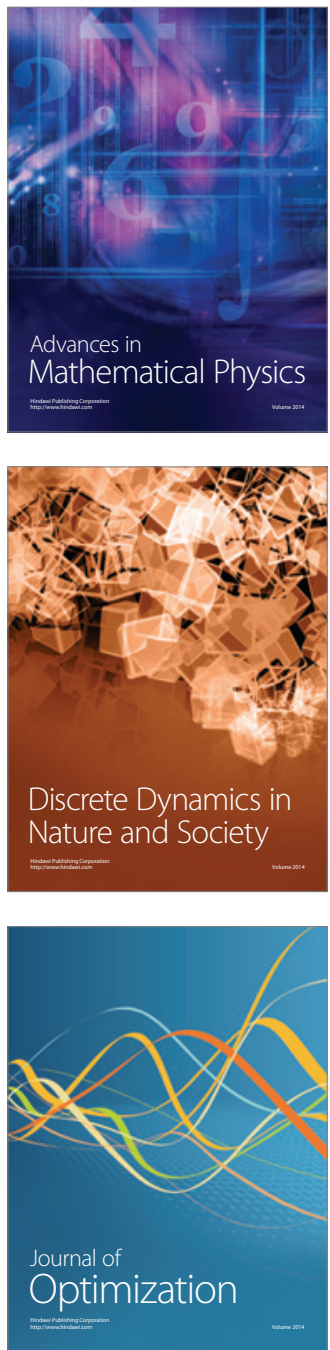